\title{
The effect of conscious controlled verbalization of a cognitive strategy on transfer in problem solving
}

\author{
MARY E. AHLUM-HEATH and FRANCIS J. DI VESTA \\ The Pennsylvania State University, University Park, Pennsylvania
}

\begin{abstract}
The effect of controlled verbalization on learning to solve complex problems was investigated. Fifty participants individually solved the six-disk Tower of Hanoi problem as a criterion task, following one of the five treatments represented in a $2 \times 2$ factorial design with an appended control group. One factor was the presence or absence of a practice series which required participants to provide verbal rationales for their moves. The other was the presence or absence of verbalization on the six-disk criterion task. The control participants performed the practice tasks and the criterion task without verbalization. Although practice tended to be more effective than no practice for improving performance, its strongest effect occurred when it was coupled with controlled verbalization. Controlled verbalization during the criterion task facilitated performance, but only for subjects who received no prior practice. It was concluded that verbalization was most helpful during the initial flexible stages of learning to solve problems before the skill had become organized. The discussion indicated that performance is facilitated by the quality and timing of the use of verbalizations rather than by the mere activity of verbalizing.
\end{abstract}

Readers who have difficulty comprehending a word, phrase, or sentence and problem solvers who have difficulty in making progress toward a solution often find it helpful to "think aloud" about the difficulty. We assume that the mere activity of verbalizing is of little value in either understanding or in problem solving unless it serves a useful processing function.

One kind of helpful verbalization in problem solving is stating a rule or giving a reason for an action. Good problem solvers state rules about an action just taken (or about to be taken) and evaluate the efficiency of their actions more than do poor problem solvers (Dorner, 1978; Gick \& Holyoak, 1980; Thorndyke \& Stasz, 1980). When solving mathematical problems that require invention, the requirement to state a goal (or goals), a plan of action for reaching the goal, and the reason why the action would help reach the goal results in a dramatic increase in invention over simply verbalizing about a feature that might enter into a successful solution (e.g., "Which one is bigger?') (Resnick \& Glaser, 1976).

On standard laboratory tasks, such as the Tower of Hanoi problem, instruction to verbalize a rule speeds up learning how to solve the problem and transfers across more complicated versions of the task (Gagne \& Smith, 1962). Gagne and Smith found verbalization to substantially affect performance on the Tower of Hanoi problem. Verbalization on the practice problems (two-, three-, four-, and five-disk problems) resulted in dramatically fewer moves on the criterial task (six-disk version) com-

Please address correspondence to: F. J. Di Vesta, Division of Counseling and Educational Psychology, 314 Cedar Building, The Pennsylvania State University, University Park, PA 16802. pared to the number of moves by subjects in noverbalization treatments. Since the number of verbalizations containing the starting move (e.g., move oddnumbered disk to goal pin), procedure (e.g., evennumbered disks move clockwise), or both were found to be correlated with efficiency in solving the criterion problem, the investigators concluded that verbalization was effective because it "somehow forced the problem solver to think."

The difficulty in "move" or "step" problems is not in the specific skills of generating each step, but in the organization of those skills to accomplish the end-state, since there is no immediately apparent relation between moves. Practice alone may reveal the relation and thereby facilitate problem solving, provided that during proceduralization and compilation of individual problemsolving skills (Neves \& Anderson, 1981), appropriate strategies are used, relevant stimuli are attended to, and relevant patterns (problem representations) are identified. Overt verbalizations during the initial stages of learning to solve a problem (i.e., before the procedural skills have been compiled into stable production systems) may have additional positive effects over mere practice, provided that verbalizations are directed meaningfully toward the goal or assist in goal reduction (see Owen \& Sweller, 1985).

At this level of analysis, stating anticipated or forthcoming moves being considered in a puzzle, such as the Tower of Hanoi which is used as the problem-solving task in this study, focuses attention on the relevant features of a given problem-state. Stating anticipated moves requires an overt commitment to a rule regarding how a move should be taken (planning) in terms of its conse- 
quences (evaluation). The problem solver is required to consider each move separately, to evaluate the alternatives available for a given problem-state, and to abandon moves that might lead to unfavorable consequences before a move is made. The overt activities of employing a means-ends analysis-explicating moves and goals, isolating appropriate feature-sets associated with a given problem-state, and inhibiting moves with potentially unsuccessful consequences-select out efficient goal-related skills for eventual compilation into production systems that achieve the goal-state. On the other hand, if problem solvers are required to verbalize internal events not ordinarily available to them, verbalizations would be expected to hinder problem solving (Norman \& Schallice, 1980).

Although means-ends strategies were examined in the present study, it should be recognized that the Tower of Hanoi puzzle can be solved by goal reduction (or rule induction). When higher order structures are understood (see Anzai \& Simon, 1979), the seemingly complex task of solving the puzzle becomes an elegantly simple one. Solving an $\mathrm{N}$-disk problem of transferring disks from Peg $A$ to $P e g C$ is the same as recursively solving an $N-1$ disk problem from Peg A to Peg B, moving the remaining disk to $P$ eg $C$ and then solving the $\mathrm{N}-1$ problem again for moving the disks from the $\mathrm{B}$ to the $\mathrm{C}$ peg (Wickelgren, 1974). Problem solvers might arrive at such recursive rules informally with repeated practice (Anzai \& Simon, 1979). However, we assume that their acquisition can be facilitated by the conscious use (through verbalizations) of means-ends strategies during the initial stages of learning to solve the problem, that is, at the time when individual skills are initially developed as separate rules (Rumelhart \& Norman, 1981). During these early stages of problem solving, there is "flexibility in interpreting and even changing rules" (Neves \& Anderson, 1981, p. 73). For the novice, informal overt practice without verbalization may provide only for inefficient covert verbalization of rules based on highly specific local moves without evaluation of their effectiveness. At best, such covert verbalizations in the initial stages of problem solving are likely to be unpredictably variable and unstandardized.

Once the processes of developing and using procedural rules become autonomous (self-regulated), as they do with repeated practice, they become less available to consciousness. Then the potential advantage of verbalizing each move at a lower level of a skill would impede efficient problem solving at a higher level by interfering with an abstract process that has become more or less automatic (Norman \& Schallice, 1980; Schneider \& Shiffrin, 1977). According to Neves and Anderson (1981), "Once composition starts, there is less chance that a small new addition will be able to compete with the larger composite rules. This is because in most versions of production systems larger rules are given a preference in application over smaller rules"' (p. 73). Upon the compilation of simple rules, the problem solver may think in terms of the more efficient, but more difficult to verbalize, higher order structures that reduce memory load (Egan \& Greeno, 1974).

Participants in the present experiment were inexperienced novices in solving the Tower of Hanoi puzzle. When performing this task in the present experiment, they were required to state and justify each move, before it was made, during practice on two-, three-, four-, and fivedisk problems and on the criterial (six-disk) problem. The hypotheses regarding performance on these tasks and implied by the rationale described above are: (1) practice, with verbalization, would facilitate solution of the six-disk problem more than would practice without verbalization or no practice; and (2) verbalization on the criterial task would facilitate performance for subjects who received no practice but would impede the performance of participants who had received practice coupled with verbalizations of a rationale for their moves.

These hypotheses were evaluated in a $2 \times 2$ design with an appended control group. One factor was practice (with verbalization) or no practice. The other factor was verbalization or no verbalization on the criterial task. The appended control group performed both practice problems and criterial task without verbalization.

\section{METHOD}

\section{Design}

The problem to be solved by the participant was the Tower of Hanoi puzzle. Criterial measures (number of moves and time to solution) were obtained on the six-disk version of the problem.

The overall design consisted of five treatment groups and was equivalent to a $2 \times 2$ factorial design with an appended control or comparison group. The between-subjects variables in the factorial design were verbalization during pretraining (verbalizing while performing a practice series of tasks vs. no practice) and verbalization on the criterial task (verbalization vs. no verbalization). The four groups were labeled as follows: verbal practice with verbal criterion (VP-VC), verbal practice with nonverbal criterion (VPNVC), no practice with verbal criterion (NP-VC), and no practice with nonverbal criterion (NP-NVC). The fifth group was employed as a comparison group. Its members received the practice and criterion tasks without being required to verbalize (nonverbal practice with nonverbal criterion; i.e., NVP-NVC).

\section{Participants}

College students $(N=50)$ enrolled in an introductory educational psychology course served as participants in the study. All were volunteers who received course credit for their participation. Each participant was randomly assigned to one of the five treatment groups. Randomization was recycled every 5 participants to assure equal numbers in each treatment. Each participant was administered the task individually. Students who had prior experience with the Tower of Hanoi problem were excluded from participation.

\section{Materials}

The Tower of Hanoi problem is simply a board with three pegs to hold six disks of diminishing diameters. The object is to move the six disks from the first peg to the third peg according to the rules described under Procedures. Timing was recorded by an electric timer accurate to $100 \mathrm{msec}$. The subject's moves were recorded by simply tabulating each move on a record sheet. 


\section{Procedures}

Each student participated individually in an experimental session that lasted for approximately $1 \mathrm{~h}$. Informed consent forms were read and signed by all participants, after which each received the following initial instructions:

This is the Tower of Hanoi puzzle. The object of the puzzle is to move the pyramid of disks from this pin (the starting pin) to this pin (the goal pin). (The experimenter at this point transferred the initial set of disks to the goal pin. The disks were then replaced at the starting position.] Now, there are two rules that you need to follow in doing this. The first rule is that you can only move one disk at a time. The second rule is that you can never place a larger disk on top of a smaller disk.

The verbalization variable was manipulated as in Gagne and Smith (1962), with slight modification. Participants in the verbalization treatments stated, prior to each move, where each disk was to be moved (an "if" statement as in "if this disk is moved from Peg $x$ to Peg $y$...'”) as well as why the location was chosen (a then statement as in "then these conditions, effects, or subgoals will be achieved"). The important facets of verbalization were: (1) it occurred before any move was taken, and (2) a reason for the move was to be stated. This manipulation was monitored carefully by the experimenter. The verbal practice component of the experiment entailed the successive solution of the two-, three-, four-, and fivedisk problems, while verbalizing. In the treatment combining verbal practice and nonverbal criterion (VP-NVC), the participant was informed that when the six-disk problem was administered, verbalization was no longer required and he/she should just go ahead with the solution as efficiently as possible.

The participants in the verbal practice and verbal criterion (VPVC) group were instructed that, throughout the sequence of problems (two-disk to six-disk problems), they would be required to state where the disk was to be moved and the reason(s) for the move prior to making each move as described above.

The no practice and verbal criterion (NP-VC) treatment was induced by simply administering the initial instructions and then presenting the participant with the six-disk problem without initial practice on the two- through five-disk problems. The participant in this treatment was instructed to verbally state the move location and reason for each move prior to making the move.

In the no practice with nonverbal criterion (NP-NVC) treatment, the participants were administered the initial instructions and then were asked to solve only the six-disk problem. There were no practice problems in this condition and verbalization was not required.

The participants in the nonverbal practice and nonverbal criterion (NVP-NVC) control group were administered the same instructions and tasks as those in the VP-VC group, except that the verbalization requirement was eliminated. The participants were instructed only that they would be presented with a sequence of problems beginning with practice on the two-disk problem, progressing through the three-, four-, and five-disk problems, and culminating with the six-disk problem.

In all treatments, the experimenter sat in view of the table and recorded the number of moves, as well as the time to solution, for the six-disk problem. All participants were required to solve each problem correctly.

\section{RESULTS}

We were interested primarily in the interaction of practice and verbalization during the criterion task. Consequently, our primary design was a $2 \times 2$ factorial analysis of variance (ANOVA) with an appended control group. In order to include the control group's results, a fivegroup, one-factor ANOVA was performed subsequent to the two-factor ANOVA. These analyses were made for each criterial measure. The overall summary of means (number of excess moves and time-to-solution) is presented in Table 1.

\section{Analysis of Excess Moves}

The six-disk problem can be solved without errors in 63 moves $\left(2^{6}-1\right)$. The number of moves in excess of that amount was the unit of analysis. Increasingly larger numbers represent correspondingly inefficient solutions characterized by trial and error or use of incorrect rules.

A $2 \times 2$ factorial ANOVA was made of the number of excess moves on the criterion task. The two betweensubjects variables in this analysis were practice (practice coupled with verbalization or no practice) and criterion task verbalization (verbalization or no verbalization). This analysis yielded a significant main effect for practice $[F(1,36)=31.69, p<.001]$, indicating that participants in the no-practice treatment made nearly four times as many excess moves (mean $=128.4$ ) as did those in the verbalized-practice treatment (mean $=34.7$ ).

The main effect for the criterion task (i.e., verbalization or no verbalization) factor was marginally significant $[F(1,36)=4.00, p=.05]$. Verbalization during performance of the criterion task led to about one-third fewer excess moves (mean $=64.9$ ) than did performance without verbalization (mean $=98.3$ ).

The most important finding is that interpretation of these significant main effects must be qualified since the interaction of practice and criterion task verbalization was also significant $[F(1,36)=10.53, p=.003]$. Follow-up analyses of the simple effects involved in this interaction were made by using Tukey's wholly significant difference (WSD) statistic (see Games, 1971). The results indicated that the NP-NVC treatment yielded significantly higher numbers of excess moves than did the NP-VC, VP-VC, and VP-NVC treatments. Although the mean number of excess moves for subjects who verbalized during practice and who also verbalized on the criterion task (VP$\mathrm{VC}$ ) is nearly double that of those who did not verbalize on the criterion task (VP-NVC), the difference was only marginally significant $(p<.10)$ via the Tukey WSD follow-up tests. Thus, performance following verbal practice was not significantly improved by verbalizing during the criterion task, although it is not safe to conclude that it was affected adversely. Nevertheless, both groups

Table 1

Summary of Means and Standard Deviations for Excess Moves and Time to Solve the Criterion Problem

\begin{tabular}{|c|c|c|c|c|}
\hline \multirow[b]{3}{*}{ Treatments } & \multicolumn{4}{|c|}{ Dependent Measures } \\
\hline & \multicolumn{2}{|c|}{ Excess Moves } & \multicolumn{2}{|c|}{ Time (Secs) } \\
\hline & Mean & $S D$ & Mean & $S D$ \\
\hline VP-NVC & 24.4 & 25.7 & 501.5 & 363.9 \\
\hline VP-VC & 45.1 & 29.6 & 874.5 & 484.6 \\
\hline NP-NVC & 172.1 & 76.1 & 1062.0 & 374.4 \\
\hline NP-VC & 84.8 & 61.6 & 1686.0 & 992.6 \\
\hline Control (NVP-NVC) & 136.0 & 82.8 & 882.5 & 764.9 \\
\hline
\end{tabular}

Note $-\mathrm{VP}=$ Verbalization with practice $; \mathrm{NP}=$ No practice; $\mathrm{VC}=$ Verbalization on criterion task; $\mathrm{NVC}=$ No verbalization on criterion task; NVP $=$ No verbalization during practice. 
exhibited significantly fewer excess moves than subjects who did not receive the series of practice problems.

The effect of practice alone (practice without verbalization) was separated from the effect of the verbalized practice treatment by a single-factor analysis of variance of the number of excess moves made by the five groups. As might be expected from the foregoing presentation of results, this analysis yielded a significant main effect due to treatments $[F(4,45)=10.57, p<.001]$. Multiple comparisons via Tukey's WSD statistic focused only on the comparison of the effects due to the NVP-NVC comparison group with the effects due to the other treatments since the remaining comparisons were represented in the previous analysis.

This analysis indicated that the NVP-NVC treatment resulted in a significantly $(p<.05)$ greater number of excess moves than both the VP-VC and the VP-NVC treatments. The other comparisons were marginally significant $(.05<p<.10)$. The NVP-NVC treatment resulted in a greater number of excess moves than the NPVC treatment but fewer excess moves than the NP-NVC moves treatment.

\section{Analysis of Time to Solution}

A $2 \times 2$ analysis of variance, parallel to that reported for the analysis of excess moves, was made of time to solution on the six-disk problem. Both of the main effects were significant in this analysis: The effect of practice yielded $F(1,36)=12.6, p<.001$, and the effect of criterion task verbalization yielded $F(1,36)=6.6, p=.04$. The interaction was not significant $(F=1.00)$.

The mean times to solve the criterial six-disk problems for each treatment are displayed in Table 1; it can be seen that verbalization coupled with practice resulted in significantly more efficient solution times than when no practice was provided. The time taken by the latter group is twice that taken by the former. Pairwise tests on solution times showed that the VP-NVC treatment required a significantly shorter time (mean $=8.4 \mathrm{~min}$ ) to complete the six-disk problem than did each of the four other treatments, which did not differ significantly from one another (combined mean $=18.8 \mathrm{~min}$ ).

The significant main effect due to verbalization on the criterion task indicates that solutions took longer when subjects had to verbalize during the criterion task than when verbalization was not required. In general, the analysis of excess moves clearly indicates that verbalization had strong effects, both during practice and during the criterial task (when the criterial task was not preceded by practice). Verbalization facilitated the initial stages of solving problems within the problem domain of the Tower of Hanoi task, and, when combined with practice, significantly improved performance whether or not the participant was required to verbalize on the criterion task. A trend in the data showed that, although practice is more effective than no practice, practice coupled with controlled verbalization leads to improved problem-solving performance.

\section{DISCUSSION}

The conscious use of the "if-then" verbalization strategy encouraged participants to control moves toward the overall goal to identify how a trial would contribute to reaching that goal, and to evaluate the effectiveness of the move after it was made. Thus, the strategy made prominent Meichenbaum's (1977) criteria for appropriate self-statements in self-regulation training of cognitive strategies (Brown, Bransford, Ferrara, \& Campione, 1983). The most important of our results indicated that the value of self-instruction depends on where it occurs in the stages of problem solving. When verbalization occurred with practice, it clearly enhanced problem solving. These results provide strong support for a declarative to procedural shift in the development of automatization (Shiffrin \& Dumais, 1981).

Individual approaches to problem solution typically reflect attempts to make the problem more manageable. Subgoal analysis, one of the ways in which problems become more manageable, has the concomitant effect of reducing the size of the problem space (Newell \& Simon, 1972). With a single exposure (i.e., the six-disk version) to the Tower of Hanoi problem, the data from the present study imply that verbalization made salient the features required for subgoal analysis that might otherwise have been unavailable (or not immediately available) to the problem solver. Through verbalizing, the problem solver could change his or her representation of the task by becoming aware of the requirements of the task and by obtaining feedback regarding the validity of a given move from anticipated consequences of that move. This explanation is consistent with Anzai and Simon's (1979) finding that a means-ends strategy may result in knowledge concerning goal and subgoal structure.

This study has demonstrated clearly that the conscious verbalization of a justification statement facilitates problem-solving performance. Most facilitation occurred in the initial phases of learning when the development of rules was presumably flexible. Conscious verbalization may then be a useful means for implementing the selfregulation of learning strategies when appropriately timed. Luria (1961) assumed overt verbalization to be an intermediate stage in the use of language for self-regulation of behavior in children on a bulb-pressing task. The results of the present study indicate that his assumptions may apply equally to adults as novices.

The further empirical examination of hypotheses offered in the introduction of the present report will extend the results of the present study to the theoretical understanding of the development of cognitive skills. The positive effect of verbalization is clearly consistent with production-system analyses of learning, an interpretation that could be made forcefully if "if-then" verbalization proves more useful than other nontrivial types of verbalizations in future studies (e.g., the examination of effects of verbalizing a review of how the problem-state after each move would help on subsequent moves). Such studies will 
help to delimit further the types, timing, and qualities of conscious verbalization of rules as they contribute to the learning automatization of a range of activities from motor to cognitive skills.

In the interest of coherence of the literature, we should also note that there is a discrepancy between the present conclusions and Sweller's (Owen \& Sweller, 1985; Sweller, Mower, \& Howe, 1982) conclusion:

It is possible that all problem-solving search activity interferes with knowledge acquisition and the amount of interference is directly related to the efficiency of the search strategy employed. Means-ends analysis, as the most efficient search strategy, may be the least efficient if scheme acquisition is desired. (Owen \& Sweller, 1985, p. 284)

Owen and Sweller (1985) indicate further that "use of reduced goal specificity procedures might be preferred in the early stages of teaching new principles"' (p. 284). This discrepancy may be due, in part, to differences in tasks. In their study, students learned to solve mathematics problems where the basic (geometric) variables and equations were known. Thus, history-cued strategies could be employed. In the present study, no simple recursive rule is readily available for the Tower of Hanoi problem. Although it can be solved by a rule-induction procedure (see introduction to this article) problem solvers in the present study, being novices, solved it by a means-ends analysis and, indeed, by use of verbalizations, were encouraged to do so.

\section{REFERENCES}

Anzai, Y., \& Simon, H. A. (1979). Theory of learning by doing. Psychological Review, 86, 124-140.

Brown, A. L., Bransford, J. D., Ferrara, R. A., \& Campione, J. C. (1983). Learning, remembering, and understanding. In J. H. Flavell \& E. M. Markman (Eds.), Handbook of child psychology: Vol. 3. Cognitive development (pp. 177-266). New York: Wiley.

DORNER, D. (1978). Theoretical advances of cognitive psychology relevant for instruction. In A. Lesgold, J. Pellegrino, S. Fokkema, \& R. Glaser (Eds.), Cognitive psychology and instruction (pp. 231-252). New York: Plenum Press.

Egan, D. E., \& Greeno, J. G. (1974). Theory of rule induction.
Knowledge acquired in concept learning, serial pattern learning, and problem solving. In L. W. Gregg (Ed.), Knowledge and cognition (pp. 43-103). Hillsdale, NJ. Erlbaum.

Gagne, R. M., \& SMith, E. C. (1962). A study of the effects of verbalization on problem solving. Journal of Experimental Psychology, 63, 12-18.

Games, P. A. (1971). Multiple comparisons of means. American Educational Research Journal, 8, 531-565.

GiCK, M. L., \& HOLYOAK, K. J. (1980). Analogical problem-solving. Cognitive Psychology, 12, 306-335.

LURIA, A. R. (1961). The role of speech in the regulation of normal and abnormal behavior. Oxford. Pergamon Press.

Meichenbaum, D. H. (1977). Cognitive behavior modification. New York. Plenum Press.

Neves, D. M., \& ANDERSON, J. R. (1981). Knowledge compilation: Mechanisms for the automatization of cognitive skills. In J. R. Anderson (Ed.), Cognitive skills and their acquisition (pp. 57-84). Hillsdale, NJ: Erlbaum.

NeWELL, A., \& SiMON, H. A. (1972). Human problem solving. Englewood Cliffs, NJ: Prentice-Hall.

Norman, D. A., \& Schallice, T. (1980). Attention to acquisition Willed and automatic control of behavior (CHIP Tech. Rep. 99). La Jolla, CA: University of California.

OWEN, E., \& SWELLER, J. (1985). What do students learn while solving mathematics problems? Journal of Educational Psychology, 77, 272-284.

Resnick, L. B., \& Glaser, R. (1976). Problem-solving and intelligence. In L. B. Resnick (Ed ), The nature of intelligence (pp. 205-230). Hillsdale, NJ. Erlbaum.

Rumelhart, D. E., \& Norman, D. A. (1981). Analogical processes in learning. In J. R. Anderson (Ed.), Cognitive skills and their acquisition (pp. 335-360). Hillsdale, NJ: Erlbaum.

SCHNEIDER, W \& SHIFFrin, R. M. (1977). Controlled and automatic human information processing: I Direction, search, and attention. Psychological Review, 84, 1-66.

Shiffrin, R. M., \& Dumais, S. T. (1981). The development of automatism. In J. R. Anderson (Ed.), Cognitive skills and their acquisition (pp. 111-140). Hillsdale, NJ: Erlbaum.

SWeller, J., Mower, R. F , \& Howe, W. (1982). Consequences of history-cued and means-ends strategies in problem solving. American Journal of Psychology, 95, 455-483.

THORNDYKe, P., STASZ, C. (1980). Individual differences in procedures for knowledge acquisition from maps. Cognitive Psychology, 12, 137-175.

Wickelgren, W. A. (1974). How to solve problems. San Francisco: Freeman.

(Manuscript received June 25, 1985; revision accepted for publication November 25,1985 .) 